\title{
Concentration of the CS-846 Epitope in Serum and Synovial Fluid of Horses with Different Grades of Osteochondral Fragments in the Carpal Joints
}

\author{
Hipolito Chavez ${ }^{1}$, Hugo Folch ${ }^{2}$, Oscar Araya ${ }^{3}$, Benjamin Uberti ${ }^{4}$ and Gabriel Moran ${ }^{4}$ \\ ${ }^{1}$ Faculty of Veterinary Medicine, Universidad San Sebastian, Concepción, Chile \\ ${ }^{2}$ Department of Immunology, Faculty of Medicine, Universidad Austral de Chile, Valdivia, Chile \\ ${ }^{3}$ Department of Clinical Veterinary Sciences, Faculty of Veterinary Sciences, Universidad Austral de Chile, Valdivia \\ ${ }^{4}$ Department of Pharmacology, Faculty of Veterinary Sciences, Universidad Austral de Chile, Valdivia, Chile
}

"Corresponding author: Gabriel Moran, Department of Pharmacology, Faculty of Veterinary Sciences, Universidad Austral de Chile, Valdivia, Chile, Tel: +56-63-221936; Fax: +56-63-221444; E-mail: gmoran@uach.cl

Rec date: March 9, 2016; Acc date: May 18, 2016; Pub date: May 24, 2016

Copyright: ( 2016 Hipolito $\mathrm{C}$, et al. This is an open-access article distributed under the terms of the Creative Commons Attribution License, which permits unrestricted use, distribution, and reproduction in any medium, provided the original author and source are credited.

\begin{abstract}
The aim of this study was to determine serum and synovial concentrations of the CS-846 epitope of articular cartilage aggrecan, in horses with a radiological diagnosis of osteochondral fragmentation (OF). For this purpose, 20 thoroughbred horses with unilateral radiocarpal or intercarpal OF were used, and 10 clinically and radiologically healthy Thoroughbred horses were assigned to the control group. Serum and synovial concentrations of the CS-846 epitope were measured in both groups by means of ELISA. The concentration of the CS-846 epitope in synovial fluid was significantly higher in carpal joints with moderate articular damage (grade 2 OF) than in the control group or in horses with severe disease (grade 3 and 4 OF). In serum, non-statistical differences in the concentration of the CS-846 epitope were observed between horses with OF and controls. We conclude that the concentration of CS-846 epitope in synovial fluid suggests an increase in cartilage aggrecan synthesis, which may be associated with the presence of moderate clinical disease. The CS-846 epitope concentration in synovial fluid may be a useful biomarker for the study of carpal OF in horses, providing a measure of the balance between cartilage synthesis and degradation in this equine disease.
\end{abstract}

Keywords: CS-846; OF; Carpal joint; Horses

\section{Introduction}

Lameness is one of the most common and important causes of failure during training of the equine athlete: athletic activity entails repetitive overloading of joints and may lead to articular cartilage damage, often accompanied by synovitis, tendon and ligament lesions and progressive subchondral bone sclerosis [1,2]. Carpal osteochondral fragments, or chip fractures, are a common cause of lameness in race horses. Osteochondral fragments are pieces of osteochondral tissue (cartilage or subchondral bone) that detach from the edges of the bones in a synovial joint. This condition occurs with high incidence in the carpal joint, and it seems most prevalent in young racehorses from 2 to 4 years of age who are undergoing rigorous athletic training [3-5]. The distolateral aspect of the radius bone, distal aspect of the radial carpal bone and proximal aspects of the intermediate and third carpal bones are common sites of OF; biomechanical loading during hyperextension of highly mobile joints explains the predominance of lesions on the dorsal articular surfaces [3,4,6-13]. Repetitive cyclic loading on the subchondral bone eventually leads to bone sclerosis and focal areas of microfracture, leading to collapse of the weakened tissue and fragmentation of cartilage and bone $[14,15]$. Such fragments may be a poor prognostic indicator for future athletic performance [15].

Articular cartilage is composed of chondrocytes embedded in an extracellular matrix of type II collagen fibrils. This matrix provides tensile strength and promotes the retention of proteoglycans (PG). Aggrecan is an important part of this matrix; it is a high molecular weight proteoglycan which bestows compressive resistance to cartilaginous tissue [16]. Molecular markers of proteoglycan origin can be released into synovial fluid (SF) and serum, and may be useful for monitoring metabolic activity (anabolism or catabolism) of cartilage over time. For example, keratan sulfate increases in synovial fluid as a marker of articular cartilage catabolism [17]. The 846 epitope of chondroitin sulfate (CS-846) is another indicator of changes in cartilage glycosaminoglycan content, specifically of aggrecan turnover, and it can be measured in SF by immunoassay [18-21]. In humans, the release of CS-846, normally present in juvenile articular cartilage but almost completely absent from adult cartilage, is closely correlated with aggrecan synthesis [22]. A previous study in horses found that CS-846 epitope increases not only in SF but also in serum of joints with OF [19]. However, CS-846 expression has not been correlated with the varying severity of osteochondral fragmentation. We hypothesize that CS-486 concentration varies depending on the severity of osteochondral lesions in joints. Thus, the objective of the present study was to determine serum and synovial concentrations of CS-846 in horses with a radiological diagnosis and different grades of disease, compared to healthy horses.

\section{Material and Methods}

\section{Horses}

Thirty thoroughbred horses were included in this study (16 females and 14 males), with a mean age of 3 years (range 2-5 years old) and a mean weight of $428 \mathrm{~kg}$ (range 390-466 kg). Twenty animals had different degrees of carpal OF, and ten healthy animals represented the 
Citation: Hipolito C, Hugo F, Oscar A, Benjamin U, Gabriel M (2016) Concentration of the CS-846 Epitope in Serum and Synovial Fluid of Horses with Different Grades of Osteochondral Fragments in the Carpal Joints. Gen Med (Los Angeles) 4: 242. doi: $10.4172 / 2327-5146.1000242$

Page 2 of 5

control group. The time of pre-existence was not determined for this study.

Owner's consent was obtained in all cases before inclusion in the study. All procedures were approved by the Bioethical Committee of the Universidad Austral de Chile.

\section{Clinical and radiographic examinations}

Physical and lameness examinations were performed on all horses before inclusion in the study. Localized lameness, heat, pain and effusion of the carpal joints was recorded in all cases. Stationary (standing) and dynamic (trotting) flexion tests were used to subjectively assess joint pain and synovial effusion. All included horses underwent carpal radiographic examinations, consisting of five radiographic views (dorsopalmar, lateromedial, dorsopalmar lateromedial oblique, dorsopalmar mediolateral oblique and flexed lateromedial) of each carpus were examined. Radiographic lesions were classified depending on the presence of osteochondral fragments and underlying bone lesions, in the following manner: grade 0: join with no evidence of osteochondral fragmentation $(n=10)$; grade 1 : minimal continuity loss of the dorsal bone surface (less than $5 \mathrm{~mm}$ ) (n $=8)$; grade $2: 45 \%$ loss of the articular surface $(n=8)$; grade $3: 60 \%$ loss of the articular surface $(n=8)$; grade 4 : significant subchondral loss with loss of support of the dorsal surface $(\mathrm{n}=8)$.

\section{Synovial fluid and serum samples}

Each study subject was sampled once. Horses were restrained manually during the collection of all samples, using a twitch when needed; sedation was not used. Whole blood samples were collected by jugular venipuncture into plain sterile tubes without anticoagulant. For serum extraction from whole blood, the tubes were stored vertically for $30 \mathrm{~min}$ at $37^{\circ} \mathrm{C}$ and then centrifuged for 7 minutes at $650 \times \mathrm{g}$. The serum was kept frozen at $-70^{\circ} \mathrm{C}$ until used.

Samples of synovial fluid were obtained by arthrocentesis using $21 \mathrm{G}$ or $18 \mathrm{G}$ sterile needles. Samples were obtained from the radiocarpal or intercarpal joints, depending on the location of the osteochondral fragments in each case, using a dorsal or dorsolateral approach. Samples were collected in dry tubes, centrifuged at $690 \times \mathrm{g}$ for 30 minutes at room temperature to remove cells, and the supernatant was stored at $-70^{\circ} \mathrm{C}$ until analyzed.

\section{Immunoassay of the CS-486 epitope}

The concentration of the CS-846 epitope in serum and synovial fluid was assessed by competitive ELISA. For this assay, we used a commercial kit (IBEX Pharmaceuticals Inc., Canada) using a mouse monoclonal anti-bovine aggrecan, which cross-reacts with equine aggrecan as described previously [19]. The serum and synovial fluid were diluted 1:5 and 1:30, respectively. The samples were added to each well of a microtiter plate that was pre-coated with goat anti-mouse immunoglobulin. Later, CS-846 epitope labeled with biotin was added, followed by a mouse IgM antibody specific for CS-846. A calibration curve was generated using a standard solution of known concentrations of bovine aggrecan. Streptavidin-peroxidase conjugate was added to each well, and then tetramethylbenzidine (TMB) substrate was added. The reaction was stopped with $\mathrm{H}_{2} \mathrm{SO}_{4}(0.2 \mathrm{M})$. Finally, the absorbance was measured in a spectrophotometer to obtain the optical density (OD), which is inversely proportional to the concentration of the epitope in the samples. The OD was converted to the concentration of CS-846 epitope $(\mu \mathrm{g} / \mathrm{ml})$ using the computer program GraphPadPrism 4.0 for non-linear calibration curves.

\section{Statistical analysis}

All statistical analyses were done with GraphPad Prism version 4.0 for Windows (GraphPad Software, La Jolla California USA). Data homoscedasticity was evaluated by Kolmogorov-Smirnov test. Differences between the mean values of the variables in horses with carpal OF and controls were evaluated by analysis of variance (ANOVA). When significant differences were obtained, we applied the method of Bonferroni-Dunn's multiple comparison test. A value of $\mathrm{P}<$ 0.05 was considered statistically significant.

\section{Results}

All data was not normally distributed. The observed concentrations of the CS-846 epitope were significantly higher $(\mathrm{P}<0.05)$ in synovial fluid than in serum, both in horses with carpal joint $O F$ and in controls. The mean serum concentrations of the CS-846 epitope were $0.64 \pm 0.50,0.40 \pm 0.33,0.26 \pm 0.33$ and $0.60 \pm 0.27(\mu \mathrm{g} / \mathrm{ml} \pm \mathrm{SD})$ for horses carrying carpal OF grades 1, 2, 3 and 4, respectively, and $0.17 \pm$ $0.13(\mu \mathrm{g} / \mathrm{ml} \pm \mathrm{SD})$ for control horses. On the other hand, the SF concentrations of the CS-846 epitope were $3.80 \pm 1.77,5.05 \pm 2.63$, $1.98 \pm 2.92$, and $1.65 \pm 0.79(\mu \mathrm{g} / \mathrm{ml} \pm \mathrm{SD})$ for horses with carpal OF grades $1,2,3$ and 4 , respectively, and $2.86 \pm 0.51(\mu \mathrm{g} / \mathrm{ml} \pm \mathrm{SD})$ for control horses. In the synovial fluid of the radiocarpal or intercarpal joints, the concentrations of the CS-846 epitope in horses with grade 2 OF were significantly higher (Figure 1).

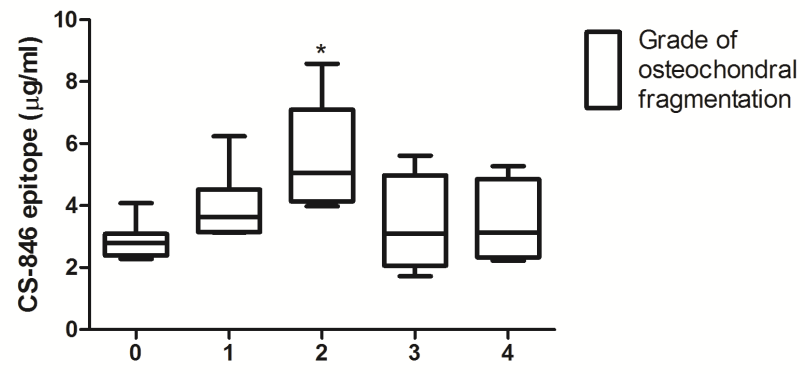

Figure 1: CS-846 epitope concentration in synovial fluid from horses with varying grades of osteochondral fragmentation (OF, grades 0 -4) in radiocarpal or intercarpal joints. Each box plot is composed of five horizontal lines displaying the 10th, 25th, 50th (median), 75th and 90th percentiles of the epitope concentration $(\mu \mathrm{g} / \mathrm{ml})$ obtained from different groups of equines. $\mathrm{A}{ }^{*}$ indicates $(\mathrm{P}$ $<0.05)$.

than in control horses or other animal groups with grades 1, 3 and 4 OF. However, the serum concentrations of the CS-846 epitope in horses with grades $1,2,3$ or 4 carpal were not significantly different (Figure 2) from one another or the control group. 
Citation: Hipolito C, Hugo F, Oscar A, Benjamin U, Gabriel M (2016) Concentration of the CS-846 Epitope in Serum and Synovial Fluid of Horses with Different Grades of Osteochondral Fragments in the Carpal Joints. Gen Med (Los Angeles) 4: 242. doi: $10.4172 / 2327-5146.1000242$

Page 3 of 5

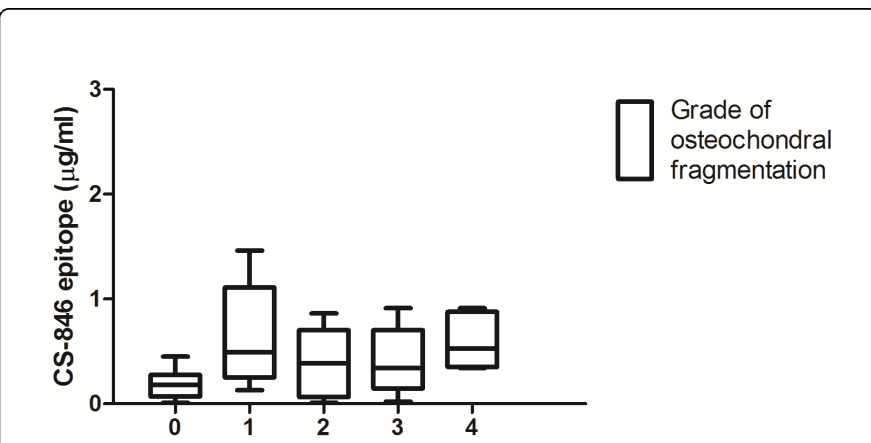

Figure 2: CS-846 epitope concentration in serum from horses with varying grades of osteochondral fragmentation (OF, grades $0-4)$ in radiocarpal or intercarpal joints. Each box plot is composed of five horizontal lines displaying the 10th, 25th, 50th (median), 75th and 90th percentiles of the epitope concentration $(\mu \mathrm{g} / \mathrm{ml})$ obtained from different groups of horses. $\mathrm{A}^{*}$ indicates $(\mathrm{P}<0.05)$.

\section{Discussion}

In this study, the highest concentrations of the CS-846 epitope were found in the SF of horses with grades 1 and 2 carpal OF. These results suggest that there may be a reparative response by the chondrocytes, because the CS-846 epitope is expressed only in new aggrecan molecules [18]. These same authors show that the CS-846 epitope is a good indicator of aggrecan synthesis, which suggests that this protein may be considered a marker of cartilage anabolic activity. Additionally, both the rate of release of the CS-846 epitope (on fragments of PG) and its concentration in the SF may indicate the rate of aggrecan synthesis [22]. In contrast, more severe joint damage (grade $4 \mathrm{OF}$ ) is associated with low biosynthetic activity by chondrocytes [23].

In horses with OF, the concentration of the CS-846 epitope was higher in the SF than in the serum. The results of this study are consistent with investigations of Frisbie et al. [19] in horses with carpal joint OF, which found higher concentrations of the CS-846 epitope in the SF than in the serum of the same animals. In turn, Matyas et al. [24], in an experimental model of osteoarthritis (OA) in dogs, also found significantly higher levels of the CS-846 epitope in the SF than in the serum. Differences in the concentration of the epitope between the SF and serum have been observed in human arthropathies as well. A study by Poole et al. [22] also found that the CS-846 epitope reached higher levels in the SF than in the serum of patients with OA or rheumatoid arthritis (RA). Thus, it seems clear that although the CS-846 epitope can occasionally be detected in blood/serum, it typically it seems to remain exclusively in the synovial cavity. Indeed, the epitope is exclusive of specific cartilage PG aggrecans, and has not been found in tissues other than articular cartilage, with the sole exception of a study by Antoniou et al. [25] which identified this epitope in the nuclei of human intervertebral discs.

The current study found a significant degree of synovitis in horses with OF. Several mechanisms could explain the different CS-846 epitope concentrations found in the SF and serum of horses with carpal joint OF. Simkin et al. [26] suggest that the number of molecules from normal cartilage detected in the SF (including fragments of PG) is determined not only by the rate of release of these molecules but also by factors affecting the clearance of molecules from the synovial cavity. These factors include the volume of the SF in the joint space, the blood flow and inflammation of the synovial membrane, joint mobility and the composition of the synovial fluid, particularly its leukocyte content [27]. As mentioned above, Myers [27] suggests that joint inflammation could alter the clearance of biological markers from joint cavities. In a follow-up study, Myers et al. [28] found an accelerated clearance of albumin-I131 in a model of canine OA with moderate synovitis, indicating that the inflamed synovial membrane might favor the clearance of low molecules but not the large PG molecules expressing the CS-846 epitope. On the other hand, older studies by Page-Thomas et al. [29] in rabbits with experimental arthritis found no significant differences between PG clearance from the inflamed synovial cavity versus controls.

Our findings show an increased concentration of CS-846 epitope in $\mathrm{SF}$ of horses with moderate (grade 2) OF. This suggests either reduced clearance or augmented release from cartilage, as explained above, and may well be related to mechanical damage of chondrocytes. Studies by Elder et al. [30] indicate that chondrocytes are sensitive to compressive forces that accumulate over time. D'Lima et al. [31] found $47 \%$ apoptosis in cell cultures of bovine chondrocytes that received pressure loads of 23 megapascals (MPa) for 48 hours. If we assume that $7 \mathrm{MPa}$ is the upper limit of physiological stress of cartilage [32], this might suggest that a stress load that causes osteochondral fracture, such as grade $4 \mathrm{OF}$, may be sufficient to induce apoptosis of these cells, which could significantly compromise the synthesis of PG. Other studies suggest that the dislocation of results in a lower loading of the cartilage, which could influence the synthetic activity of the chondrocytes [33]. Those authors showed that chondrocytes in the cartilage from the part of the fractured bone that continued to bear a load did not express cartilage oligomeric matrix protein (COMP) mRNA, which suggests that the reduced mechanical compression of the dislocated OF stimulates chondrocyte synthetic activity, possibly contributing to the up-regulation of the CS-486 epitope.

The low concentrations of the CS-846 epitope that were recorded in the SF of horses with grades 3 and 4 carpal OF may be associated with the marked inflammatory process occurring in these degrees of injury. Inflammation may inhibit the synthesis of aggrecan through excessive production of pro-inflammatory cytokines [34]. Chondrocytes express several chemokines and have receptors that enable responses associated with cartilage catabolism [35-37]. Both in vitro and in vivo studies indicate that interleukin-1 (IL-1) and tumor necrosis factor (TNF)- $\alpha$ are the predominant catabolic cytokines involved in the initiation and progression of articular cartilage destruction and that they have an adverse effect on the synthesis of aggrecan by chondrocytes [38,39]. In addition to inducing the synthesis of metalloproteinases and other proteinases by chondrocytes, IL-1 and TNF- $\alpha$ increase the synthesis of prostaglandin E2 (PGE2) by stimulating the expression or activities of COX-2, microsomal PGE synthase-1 (mPGES-1) and soluble phospholipase A2 (sPLA2). They also up-regulate the production of nitric oxide via inducible nitric oxide synthetase (iNOS, or $\mathrm{NOS}_{2}$ ), other proinflammatory cytokines, such as IL-6 [40], leukemia inhibitory factor (LIF), IL-17 and IL-18, and chemokines, such as IL-8 [38]. The extent of inflammation can vary widely depending on the amount of mechanical damage to chondrocytes, and thus, it seems that marked inflammation in severe clinical cases may actually lead to reduced synthesis of aggrecan and less CS-846 concentration in SF of affected joints.

In conclusion, our findings showed increased concentration of CS-846 epitope in synovial fluid of horses with moderate (grade 2), 
Citation: Hipolito C, Hugo F, Oscar A, Benjamin U, Gabriel M (2016) Concentration of the CS-846 Epitope in Serum and Synovial Fluid of Horses with Different Grades of Osteochondral Fragments in the Carpal Joints. Gen Med (Los Angeles) 4: 242. doi: $10.4172 / 2327-5146.1000242$

Page 4 of 5

suggesting an increase in cartilage aggrecan synthesis compared to healthy joints $[41,42]$. The lack of a significant increase of CS-846 epitope concentration in joint fluid of horses with more severe (grade 3 and 4) may suggest decreased aggrecan synthesis due to severe inflammation. The CS-846 epitope in synovial fluid may be a useful biomarker in the study of carpal OF in horses. Further studies are needed to determine whether this epitope may provide a measure of the balance between cartilage synthesis and degradation in osteochondral disease in horses $[43,44]$.

\section{Acknowledgments}

This work was supported by Graduate School, Faculty of Veterinary Science, Universidad Austral de Chile.

\section{References}

1. Bramlage LR, Schneider RK, Gabel AA (1988) A clinical perspective on lameness originating in the carpus. Equine Vet J Suppl 12-18.

2. Norrdin RW, Kawcak CE, Capwell BA, McIlwraith CW (1998) Subchondral bone failure in an equine model of overload arthrosis. Bone 22: 133-139.

3. Mcllwraith CW, Yovich JV, Martin GS (1987) Arthroscopic surgery for the treatment of osteochondral chip fractures in the equine carpus. J Am Vet Med Assoc 191: 531-540.

4. Stephens PR, Richardson DW, Spencer PA (1988) Slab fractures of the third carpal bone in standardbreds and thoroughbreds: 155 cases (1977-1984). J Am Vet Med Assoc 193: 353-358.

5. Secombe CJ, Firth EC, Perkins NR, Anderson BH (2002) Pathophysiology and diagnosis of third carpal bone disease in horses: A review. NZ Vet J 50: 2-8.

6. Lindsay WA, Horney FD (1981) Equine carpal surgery: A review of 89 cases and evaluation of return to function. J Am Vet Med Assoc 179: 682-685.

7. Fischer AT, Stover SM (1987) Sagittal fractures of the third carpal bone in horses: 12 cases (1977-1985). J Am Vet Med Assoc 191: 106-108.

8. Martin GS, Haynes PF, McClure JR (1988) Effect of third carpal slab fracture and repair on racing performance in thoroughbred horses: 31 cases (1977-1984). J Am Vet Med Assoc 193: 107-110.

9. Raidal SL, Wright JD (1996) A retrospective evaluation of the surgical management of equine carpal injury. Aust Vet J 74: 198-202.

10. Park RD, Morgan JP, O'Brien T (1970) Chip fractures in the carpus of the horse: a radiographic study of their incidence and location. J Am Vet Med Assoc 157: 1305-1312.

11. Palmer SE (1986) Prevalence of carpal fractures in thoroughbred and standardbred racehorses. J Am Vet Med Assoc 188: 1171-1173.

12. Schneider RK, Bramlage LR, Gabel AA, Barone LM, Kantrowitz BM (1988) Incidence, location and classification of 371 third carpal bone fractures in 313 horses. Equine Vet J Suppl 6: 33-42.

13. Johnston C, Roepstorff L, Drevemo S, Kallings P (1996) Kinematics of the distal hindlimb during stance phase in the fast trotting standardbred. Equine Vet J 28: 263-268.

14. Levick JR, Thompson PW (1988) Intra-articular volume as an important factor governing macromolecular half life in synovial fluid. Ann Rheum Dis 47: 701-702.

15. McIlwraith CW (2002) Arthroscopic surgery for osteochondral chip fragments and other lesions not requiring internal fixation in the carpal and fetlock of the equine athlete: What have we learned in 20 years? Clin Tech Equine Prac 1: 200-210.

16. Poole AR, Ionescu M, Fitzcharles MA, Billinghurst RC (2004) The assessment of cartilage degradation in vivo: development of an immunoassay for the measurement in body fluids of type II collagen cleaved by collagenases. J Immunol Methods 29: 145-53.
17. Todhunter RJ, Yeager AE, Freeman KP, Parente EJ, Lust G (1993) Keratan sulfate as a marker of articular cartilage catabolism and joint treatment in ponies. Am J Vet Res 54: 1007-1016.

18. Rizkalla G, Reiner G, Bogoch, Poole AR (1992) Studies of the articular proteoglycan aggrecan in health and osteoarthritis. J Clin Invest 90: 2268-2277.

19. Frisbie DD, Ray CS, Ionescu M, Poole AR, Chapman PL, et al. (1999) Measurement of synovial fluid and serum concentrations of the 846 epitope of chondroitin sulfate and of carboxy propeptides of type II procollagen for diagnosis of osteochondral fragmentation in horses. Am J Vet Res 60: 306-309.

20. Laverty S, Ionescu M, Marcoux M, Boure L, Doize B, et al. (2000) Alterations in cartilage type-II procollagen and aggrecan contents in synovial fluid in equine osteochondrosis. J Orthop Res 18: 399-405.

21. Robion FC, Doizé B, Bouré L, Marcoux M, Ionescu M, et al. (2001) Use of synovial fluid markers of cartilage synthesis and turnover to study effects of repeated intra-articular administration of methylprednisolone acetate on articular cartilage in vivo. J Orthop Res 19: 250-258.

22. Poole AR, Ionescu M, Swan A, Dieppe PA (1994) Changes in cartilage metabolism in arthritis are reflected by altered serum and synovial fluid levels of the cartilage proteoglycan aggrecan. Implications for pathogenesis. J Clin Invest 94: 25-33.

23. Gray ML, Pizzanelli AM, Grodzinsky AJ, Lee RC (1988) Mechanical and physiochemical determinants of the chondrocyte biosynthetic response. J Orthop Res 6: 777-792.

24. Matyas JR, Atley L, Ionescu M, Eyre DR, Poole AR (2004) Analysis of cartilage biomarkers in the early phases of canine experimental osteoarthritis. Arthritis Rheum 50: 543-552.

25. Antoniou J, Steffen T, Nelson F, Winterbotton N, Hollander AP, et al. (1996) The human lumbar intervertebral disc. Evidence for changes in the biosynthesis and denaturation of the extracellular matrix with growth, maturation, ageing, and degeneration. J Clin Invest 98: 996 - 1003.

26. Simkin PA, Bassett JE (1995) Cartilage matrix molecules in serum and synovial fluid. Curr Opin Rheumatol 7: 346-351.

27. Myers SL, O'Connor BL, Brandt KD (1996) Accelerated clearance of albumin from the osteoarthritic knee: implications for interpretation of concentrations of "cartilage markers" in synovial fluid. J Rheumatol 23: 1744-1748.

28. Myers SL, O'Connor BL, Brandt KD (1995) Effect of synovial fluid hyaluronan on the clearance of albumin from the canine knee. Ann Rheum Dis 54: 433-434.

29. Page-Thomas DP, Bard D, King B, Dingle JT (1987) Clearance of proteoglycan from joint cavities. Ann Rheum Dis 46: 934-937.

30. Elder SH, Goldstein SA, Kimura JH, Soslowsky LJ, Spengler DM (2001) Chondrocyte differentiation is modulated by frequency and duration of cyclic compressive loading. Ann Biomed Eng 29: 476-482.

31. D'Lima DD, Hashimoto S, Chen PC, Colwell CW, Lotz MK (2001) Cartilage injury Impact of mechanical trauma on matrix and cells. Clin Orthop and Relat Res 391S: 90-99.

32. von Eisenhart R, Adam C, Steinlechner M (1999) Quantitative determination of joint incongruity and pressure distribution during simulated gait and cartilage thickness in the human hip joint. J Orthop Res 17: 532-539.

33. Skiöldebrand E, Heinegard D, Eloranta ML, Nilsson G, Dudhia J, et al. (2005) Enhanced concentration of COMP (cartilage oligomeric matrix protein) in osteochondral fractures from racing thoroughbreds. J Orthop Res 23: 156-163.

34. Arend WP (2001) Cytokines and cellular interactions in inflammatory synovitis. J Clin Invest 107: 1081-1082.

35. Richardson DW, Dodge GR (2000) Effects of interleukin-1beta and tumor necrosis factor-alpha on expression of matrix-related genes by cultured equine articular chondrocytes. Am J Vet Res 61: 624-630.

36. Trumble TN, Trotter GW, Oxford JRT, McIlwraith CW, Cammarata S, et al. (2001) Synovial fluid gelatinase concentrations and matrix 
Citation: Hipolito C, Hugo F, Oscar A, Benjamin U, Gabriel M (2016) Concentration of the CS-846 Epitope in Serum and Synovial Fluid of Horses with Different Grades of Osteochondral Fragments in the Carpal Joints. Gen Med (Los Angeles) 4: 242. doi: $10.4172 / 2327-5146.1000242$

Page 5 of 5

metalloproteinase and cytokine expression in naturally occurring joint disease in horse. Am J Vet Res 62: 1467-1477.

37. Borzì RM, Mazzetti I, Marcu KB, Facchini A (2004) Chemokines in cartilage degradation. Clin Orthop Relat Res 427: 53-61.

38. Goldring SR, Goldring MB (2004) The role of cytokines in cartilage matrix degeneration in osteoarthritis. Clin Orthop Relat Res 427: S27-36.

39. Guilak F, Fermor B, Keefe FJ, Kraus VB, Olson SA, et al. (2004) The role of biomechanics and inflammation in cartilage injury and repair. Clin Orthop Relat Res 423: 17-26.

40. Gibson KT, Hodge H, Whittem T (1996) Inflammatory mediators in equine synovial fluid. Aust Vet J 73: 148-151
41. Glant TT, Mikecz K, Roughley PJ, Buzas E, Poole AR (1986) Age-related changes in protein-related epitopes of human articular cartilage proteoglycans. Biochem J 236: 71-75.

42. Kraus VB, Huebner JL, Fink C, King JB, Brown S, et al. (2002) Urea as a passive transport marker for arthritis biomarker studies. Arthritis Rheum 46: 420-427.

43. Lohmander LS, Ionescu M, Jugessur H, Poole AR (1999) Changes in joint cartilage aggrecan after knee injury and in osteoarthritis. Arthr Rheum 42: 534-544.

44. Müller-Gerbl M (1998) The subchondral bone plate. Adv Anat Embryol Cell Biol 141: 1-134. 